\title{
ESTUDO DA ESTABILIDADE, COMPORTAMENTO REOLÓGICO E EFEITO DA TEMPERATURA DA POLPA DE CUPUAÇU (THEOBROMA GRANDINFLORUM) EM DIFERENTES CONCENTRAÇÕES
}

\author{
S.F.BORGES ${ }^{1}$, V.P. PIRES ${ }^{1}$, H.A.VILLA-VELEZ ${ }^{1}$, A.A.SANTANA ${ }^{1}$, R.M.SAMPAIO ${ }^{1}$ \\ ${ }^{1}$ Universidade Federal do Maranhão, Departamento de Engenharia Química \\ E-mail para contato: simara_f.borges_@hotmail.com
}

\begin{abstract}
RESUMO - O cupuaçu (Theobroma grandinflorum) é uma fruta com alta demanda comercial nas Regiões Norte de Nordeste do Brasil. No entanto, sua industrialização é recente e pouco se conhecem sobre as características físicas da polpa e de seus coprodutos. Assim, este trabalho de pesquisa tem como objetivo estudar o comportamento reológico da polpa de cupuaçu em função do teor de sólidos solúveis totais $\left(2,6\right.$ e $\left.12{ }^{\circ} \mathrm{Brix}\right)$ e temperatura $\left(10,35\right.$ e $\left.60{ }^{\circ} \mathrm{C}\right)$. As medidas experimentais foram realizadas em viscosímetro Brookfield e o modelo reológico de Ostwald-de-Waelle foi ajustado aos dados experimentais de viscosidade aparente e taxa de deformação. O modelo utilizado teve um bom ajuste aos dados experimentais. A polpa de cupuaçu apresentou tendência de diminuição da viscosidade aparente com o aumento da taxa de deformação, comportando-se como fluído não-newtoniano com caráter pseudoplástico. Além disso, analizou-se ainda a energia de ativação comprovando-se que a equação de Arrhenius pode ser utilizada no estudo da influência da temperatura da polpa em questão. Finalmente, fez-se o estudo de estabilidade da polpa in natura.
\end{abstract}

\section{INTRODUÇÃO}

As frutas tropicais vêm trazendo grande destaque na indústria de alimentos, em grande parte, pelas características de sabor, aroma e possibilidades de utilização doméstica e agroindustrial da sua polpa. Nesse contexto, o cupuaçuzeiro (theobroma grandinflorum), que tem como fruto o cupuaçu, nativo da Amazônia Oriental, tem sido destaque dentre tais frutas (Godim et al., 2001).

Sabendo da enorme importância econômica do cupuaçu, fica evidente a necessidade de se conhecer ainda mais sobre suas propriedades reológicas a fim de se melhorar os futuros empreendimentos agroindustriais. A Reologia é a ciência que estuda a resposta de um material à aplicação de uma tensão ou deformação externa (Toledo, 1991). Sendo assim para a indústria de alimentos, o estudo reológico é de grande utilidade na determinação de cálculos em processos de engenharia, tais como cálculo de vazões, seleção de bombas, determinação de perda de carga em tubulações, em operações unitárias como evaporação e esterilização entre outros, determinação da funcionalidade de um ingrediente no desenvolvimento de um produto, testes de tempo-de-prateleira, avaliação da textura dos alimentos para correlacioná-la à análise sensorial (Holdsworth, 1993). 
Mediante o exposto, neste trabalho objetivou-se estudar o comportamento reológico da polpa de cupuaçu (theobroma grandinflorum), com diferentes teores de sólidos solúveis totais e em diferentes temperaturas e assim caracterizá-la como newtoniana ou não-newtoniana o que será feito observando a forma como sua viscosidade se comporta quando submetida a diferentes deformações, e ainda a estabilidade da polpa. Assim, tal estudo contribuirá para complementar os estudos já relatados sobre polpa de cupuaçu e em conseqüência, com a melhora em seu processamento.

\section{MATERIAL E MÉTODOS}

\subsection{Matéria prima e preparação das amostras}

Na pesquisa foi utilizada polpa de cupuaçu adquirida em um mercado local da cidade de São Luís - MA. Assim, a polpa in natura sem adição de água ou de conservantes foi medida inicialmente com um refratômetro para determinar a concentração de sólidos solúveis $\left(12^{\circ}\right.$ Brix $)$. A partir desse valor definiu-se o teor de água das polpas concentradas utilizadas no estudo, que foram de 50 e $75 \%$, correspondentes, respectivamente, ao teor de sólidos solúveis totais de 2 e $6{ }^{\circ}$ Brix.

\subsection{Estudo reológico e modelagem matemática}

A polpa de cupuaçu às diferentes concentrações de sólidos solúveis totais $(2,6$ e 10 ${ }^{\circ}$ Brix) foram submetidas a análise do comportamento nas temperaturas de 10,35 e $60{ }^{\circ} \mathrm{C}$ e taxas de deformação na faixa de 0,333 a $3,333 \mathrm{~s}^{-1}$. Na experimentação utilizou-se um viscosímetro BROOKFIELD (modelo DV-II + Pro, Brookfield, USA), utilizando um spindle LV3(63) e acoplado a um banho termostático (modelo ALB 250 C, Tecnal, Brasil).

Os reogramas obtidos foram ajustados com o modelo reológico de Ostwald-de-Waele (Lei da Potência), de acordo com a Eq. (1):

$\mu=k \dot{\gamma}^{(\eta-1)}$

Onde, $\mu$ é a viscosidade dinâmica (Pa.s), $\eta$ é o índice de comportamento (adimensional), $\gamma$ é a taxa de deformação $\left(\mathrm{s}^{-1}\right)$ e $k$ é o índice de consistência (Pa.s) (Steffe, 1996; Genovese et al., 2007).

O modelo foi ajustado aos valores experimentais de viscosidade aparente em função da taxa de deformação, utilizando-se para a modelagem matemática a ferramenta Solver do software Microsoft Excel (Microsoft, Redmond, EUA). Foram utilizados como critério de determinação de melhor ajuste do modelo aos dados experimentais o coeficiente de determinação $\left(R^{2}\right)$ e o Erro Relativo Médio $(M R E, \%)$ Eq.(2).

$M R E=\frac{100}{n} \sum_{i=1}^{m} \frac{\left|Y_{i}-Y_{i}^{*}\right|}{Y_{i}^{*}}$

Onde, $Y$ e $Y^{*}$ representam os valores experimentais e calculados, respectivamente e $m$ é o número de valores experimentais. 
Para a avaliação do efeito da temperatura sobre os valores da viscosidade aparente da polpa de cupuaçu foi utilizada a equação de Arrhenius (Eq. (3)), onde tem-se o valor da viscosidade aparente (Pa.s) em função do inverso da temperatura (1/T).

$\eta=A_{0} \exp \left(\frac{E_{a}}{R(T+273.15)}\right)$

\subsection{Estabilidade física}

A polpa de cupuaçu foi diluída na proporção de $1: 4$ e homogeneizada a $25{ }^{\circ} \mathrm{C}$ em homogeneizador Ultra Turratec (modelo TE-102, Tecnal, Brasil), usando rotações de 0 a 2000 rpm durante 10 minutos. Em seguida foi submetida à centrifugação durante $10 \mathrm{~min}$. A absorbância do sobrenadante foi determinada a $660 \mathrm{~nm}$ utilizando o espectrofotômetro. A turbidez foi determinada pela quantidade de luz absorvida pelas partículas suspensas, assim, maiores leituras de absorbância correspondem a uma maior turbidez (Okoth et al., 2000).

\section{RESULTADOS, TRATAMENTO ESTATÍSTICO E DISCUSSÕES}

\subsection{Reologia da polpa}

Na tabela 1 têm-se os valores dos parâmetros do modelo reológico de Ostwald-deWaelle, ajustados aos dados experimentais nos teores de sólidos solúveis totais $(2,6$ e 12 ${ }^{\circ}$ Brix) e nas temperaturas de 10,35 e $60{ }^{\circ} \mathrm{C}$. Para a polpa de cupuaçu obteve-se valores de $R^{2}$ acima de 0,91 e $M R E$ inferiores a $10 \%$, indicando que o modelo em questão pode ser utilizado para estimar os dados reológicos da polpa de cupuaçu nos Brix e nas temperaturas do estudo. Ao fazer a análise reológica da polpa de cupuaçu de 0,3 a $100 \mathrm{rpm}$ e em temperaturas de 20 a $70{ }^{\circ} \mathrm{C}$, Ferreira et al. (2008) constatou que o modelo que melhor se ajustou aos dados experimentais foi também o de Ostwald-de-Waelle, apresentando valores de $R^{2}$ acima de 0,94 .

Tabela 1 - Parâmetros do modelo de Ostwald-de-Waelle.

\begin{tabular}{cccccc}
${ }^{\circ}$ Brix & Temperatura $\left({ }^{\circ} \mathbf{C}\right)$ & $\boldsymbol{k}$ (Pa.s) & $\boldsymbol{\eta}$ & $\boldsymbol{R}^{\mathbf{2}}$ & $\boldsymbol{M R E}(\boldsymbol{\%})$ \\
2 & 10 & 82,28 & 0,306 & 0,97 & 3,57 \\
& 35 & 69,70 & 0,303 & 0,97 & 4,11 \\
& 60 & 73,45 & 0,087 & 0,99 & 7,83 \\
6 & 10 & 1139,90 & 0,296 & 0,99 & 3,09 \\
& 35 & 1244,00 & 0,107 & 0,99 & 2,48 \\
\multirow{2}{*}{12} & 60 & 859,60 & 0,295 & 0,99 & 3,95 \\
& 10 & 7900,00 & 0,216 & 0,99 & 7,49 \\
& 35 & 6583,34 & 0,246 & 0,99 & 1,42 \\
& 60 & 4801,40 & 0,210 & 0,99 & 1,88 \\
\hline
\end{tabular}

O índice de comportamento $(\eta)$ mostrou valores de $0,05<\eta<0,59$, caracterizando à polpa como fluido pseudoplástico. Ferreira et al. (2008) também trabalhando com polpa de 
cupuaçu integral de 0,3 a $300 \mathrm{~s}^{-1}$, encontrou valores de $\eta$ entre 0,23 e 0,42 em temperaturas de 10 a $60{ }^{\circ} \mathrm{C}$. Cabral et al. (2002) em seu estudo com polpa de cupuaçu peneirada também obteve comportamento não-newtoniano de caráter pseudoplástico.

Para o índice de consistência $k$ também se obteve variação com a concentração de sólidos solúveis totais e com a temperatura. Observou-se que o $k$ diminuiu com o aumento da temperatura tendo os maiores valores para a temperatura de $10{ }^{\circ} \mathrm{C} . \mathrm{O}$ índice de consistência também aumentou com a concentração de sólidos solúveis totais, assim obteve-se o maior índice para a concentração de $12^{\circ}$ Brix. Sousa et al. (2014) também obteve a mesma tendência do índice de consistência ao fazer a análise reológica da polpa de pequi nos teores de sólidos solúveis totais de $6,8,10$ e $12{ }^{\circ}$ Brix, e nas temperaturas de $25,30,35,40,45$ e $50{ }^{\circ} \mathrm{C}$.

Para todas as temperaturas e concentrações houve um decréscimo da viscosidade com o aumento da taxa de deformação (Figura 1). Estes resultados podem ser atribuídos ao maior alinhamento das partículas na direção da tensão aplicada que tornam a resistência ao movimento cada vez menor. Além disso, percebeu-se ainda uma diminuição da viscosidade com o aumento da temperatura e um aumento da viscosidade com o aumento no teor de sólidos solúveis totais. Assim, obtiveram-se as maiores viscosidades, experimentais e teóricas, para a concentração de $12^{\circ}$ Brix e temperatura de $10^{\circ} \mathrm{C}$. Autores como Ferreira et al. (2008), Cepeda e Villarán (1991), Ferreira et al., (2002) e Assis et al., (2006) também obtiveram resultados semelhantes em relação ao efeito da temperatura e da concentração na viscosidade aparente de polpas de frutas.

Figura 1 - Resultados experimentais da viscosidade aparente para as temperaturas de $10^{\circ} \mathrm{C}$ $(\Lambda), 35^{\circ} \mathrm{C}(\square)$ e $60^{\circ} \mathrm{C}(\bullet)$ e concentração $6^{\circ}$ Brix, Linhas (---) indicam valores calculados pelo modelo Lei da Potência.

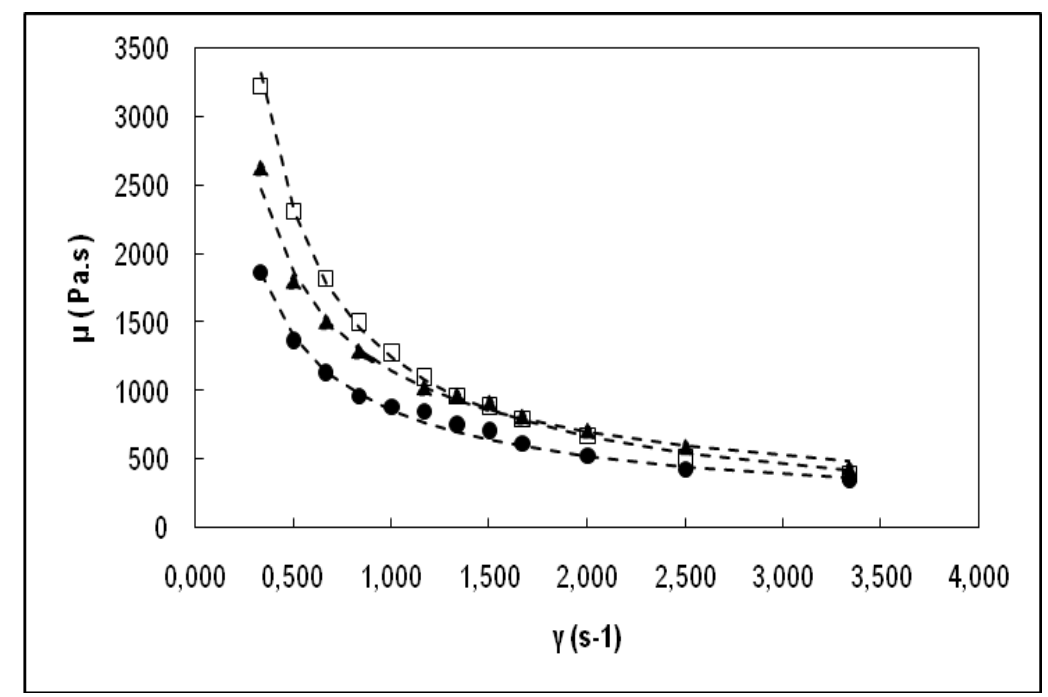

As energias de ativação mostraram tendência de redução com o aumento dos sólidos solúveis totais como mostrado na Tabela (2). Os valores de energia de ativação encontrados nesse estudo foram similares aos encontrados por Alexandre (2002), que ao analisar o comportamento reológico da polpa de açaí integral, encontrou o valor igual a $6,28 \mathrm{~kJ} / \mathrm{mol}$, Ferreira et al. (2002), que estudou o comportamento reológico da polpa de goiaba integral $(6,11 \mathrm{~kJ} / \mathrm{mol})$ e Pereira et al. (2008) que analisou a polpa de umbu $(6,15 \mathrm{~kJ} / \mathrm{mol})$. 
Tabela 2 - Energias de ativação para a polpa de cupuaçu.

\begin{tabular}{ccc|}
\hline${ }^{\circ}$ Brix & Ea (J/mol) & $\boldsymbol{R}^{\mathbf{2}}$ \\
$\mathbf{2}$ & 2393,49 & 0,98 \\
$\mathbf{6}$ & 24374,21 & 0,97 \\
$\mathbf{1 2}$ & 3772,19 & 0,99 \\
\hline
\end{tabular}

\subsection{Análise de turbidez}

A Figura 2 mostra a relação quase linear da turbidez com a velocidade de homogeneização. A turbidez variou de 0,3 a 1.3 de absorbância. Pode-se inferir a partir do gráfico que a turbidez é diretamente proporcional a velocidade de homogeneização da polpa de cupuaçu em estudo. Tal comportamento pode ser explicado pelas observações de Binner $e t$ al. 2000, onde a desintegração mecânica das partículas por aplicação de agitação a alta velocidade faz com que aumente a turbidez da polpa. Os valores de turbidez encontrados neste estudo foram próximos aos encontrados por Amstalden (1992) para suco concentrado de laranja.

Figura 2-Gráfico da turbidez da polpa de cupuaçu a $660 \mathrm{~nm}$

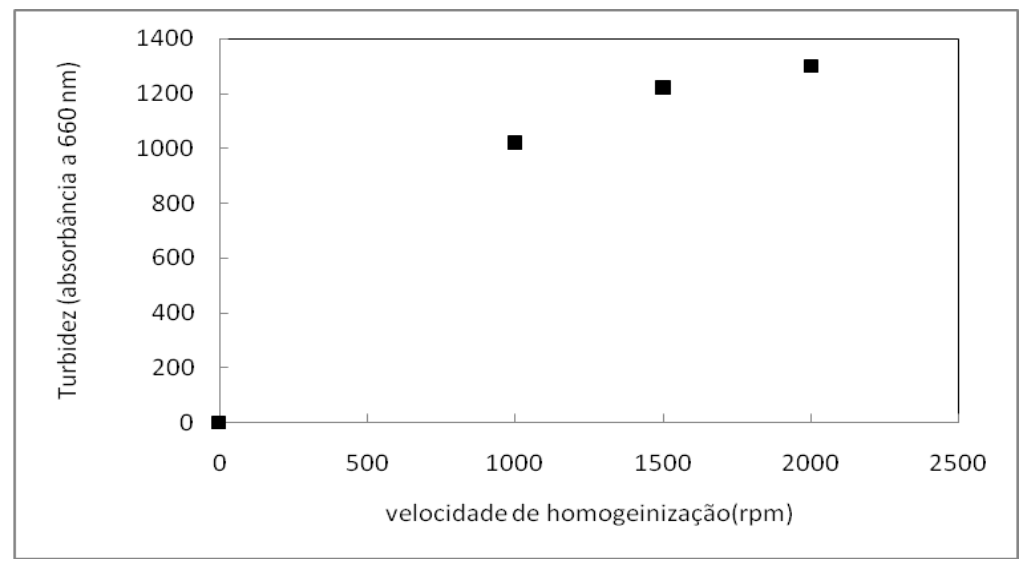

\section{CONCLUSÃO}

A polpa de cupuaçu do estudo apresentou comportamento pseudoplástico o qual foi determinado atraves do modelo de Ostwald-de-Waelle. A equação de Arrhenius na taxa de deformação estudada pode ser utilizada para expressar a influência da temperatura sobre a viscosidade aparente da polpa de cupuaçu. O estudo da estabilidade foi realizado por meio dos testes de turbidez. Os resultados demonstraram que a turbidez aumentou com o aumento da velocidade de homogeneização.

\section{REFERÊNCIAS}


ALEXANDRE, D. Conservação da polpa de açaí através da tecnologia de obstáculos e caracterização reológica. 149f. Dissertação (Mestrado em Engenharia de Alimentação) - Faculdade de Engenharia de Alimentos, UNICAMP, Campinas, 2002.

AMSTALDEN, L.C. Estudo sobre a ação da pectinesterase em suco de laranja. 200f. Dissertação (Mestrado em Engenharia de Alimentos) - Faculdade de Engenharia de Alimentos, UNICAMP, Campinas, 1992.

ASSIS, M.M. M; LANNES, SC; TADINI, C.C.; TELIS, V.R.N.; ROMERO, J.T. Influence of temperatura and concentration on thermophycal properties of yellow mombin (Spondias mombin, L.). Eur. Food Restechnol, v.223, p.585-593, 2006.

BINNER, S.; JARDINE W.G.; RENARD, C.M.C.G.; JARVIS, M.C. Cell wall modifications during cooking os potatoes and sweet potatoes. Journal of Science of Food na Agriculture, v.80, n.2, p.216-218, 2000.

CABRAL, M.F.P.; QUEIROZ, A.J.M. FIGUEIRÊDO, R.M.F. Comportamento reológico da polpa de cupuaçu (Theobroma grandiflorum schum.) peneirada. Revista Brasileira de Produtos Agroindustriais, Campina Grande, v.4, n.1, p.37-40, 2002.

CEPEDA, E.; VILLARÁN, M.C. Density and viscosity of Malus floribunda juice as a function of concentration and temperature. Journal of Food Engineering, v.41, p.103107, 1991.

FERREIRA, G. M.; GUIMARÃES, M. J. O. C.; MAIA, M. C. A. Efeito da Temperatura e Taxa de Cisalhamento nas Propriedades de Escoamento da Polpa de Cupuaçu (T.grandifl orum Schum) integral. Revista Brasileira de Fruticultura, Jaboticabal, v. 30, n. 2, p. 385-389, 2008

FERREIRA, G.M.; QUEIROZ, A.J.M.; CONCEIÇÃO, R.S.; GASPARETTO, C.A. Efeito da temperatura no comportamento reológico das polpas de caju e goiaba. Revista Ciências Exatas e Naturais, v. 4, p.175-184, 2002.

GENOVESE, D.B.; LOZANO,J.E.; RAO,M.A.The rheology of colloidal and noncolloidal food dispersions.[s.n]. v. 72.Journal of Food Science, p. 11-20, 2007.

GODIM, T.M. S; TOMAZINI, M.J; CAVALCANTE, M.J. B; SOUZA, J.M.L. Aspectos da produção de cupuaçu. Embrapa, Rio Branco, dezembro, 2001.

HOLDSWORTH, S. D. Rheological models used for the prediction of the flow properties of food products: a literature review. Trans. I. Chem. E., Part. C, p.139-78, 1993.

OKOTH, M.W. KAAHWA, A.R. IMUNGI, J.K.The effectofhomogenization, stabilizer and amylase on cloudiness of passion fruit juice. [s.n] v. 11, FoodControl, 2000, p. 305-311. Oscillatory shear behavior of umbu pulp. Revista Brasileira de Engenharia Agrícola e Ambiental, v.12, n.1, p.87-90, 2008.

PEREIRA, E.A.; BRANDÃO, E.M.; BORGES, S.V.; MAIA, M.C.A. Influence of concentration on the steady and oscillatory shear behavior of umbu pulp. Revista Brasileira de Engenharia Agrícola e Ambiental, v.12, n.1, p.87-90, 2008.

SOUSA, E. P.; QUEIROZ, A.J.M.; FIGUEIRÊDO, R.M. F. LEMOS D.M. Comportamento reológico e efeito da temperatura da polpa de pequi em diferentes concentrações. Braz. J. Food Technol.,Campinas, v. 17, n. 3, p. 226-235, jul./set. 2014.

STEFFE, F.L. Rheological methods in food process engineering. 1 ed. Freeman Press;East Lansing, 1996.

TOLEDO, R. T. Fundamentals of Food Process Engineering. New York: Chapman \& Hall, 1991., 602p. 